\title{
The da Vinci Xi System in Robotic Gastric Cancer Surgery: A Comparison with the S System
}

\author{
Chulhyo Jeon, M.D., Ho Seok Seo, M.D., Yoon Ju Jung, M.D., Cho Hyun Park, M.D., Ph.D., Kyo Young Song, M.D., Ph.D. \\ Division of Gastrointestinal Surgery, Department of Surgery, College of Medicine, The Catholic University of Korea, Seoul, Korea
}

Purpose: The next-generation da Vinci system, the Xi, was introduced in 2015. Its benefits for patients with gastric cancer have not yet been clearly identified.

Methods: A total of 47 patients who had undergone robot-assisted gastrectomy (RAG) for gastric cancer were analyzed retrospectively. Twenty-six (26) patients had undergone RAG by da Vinci S, and the remaining 21 by da Vinci Xi. These two groups' clinicopathological characteristics, operative details, and short-term postoperative outcomes were compared.

Results: The median docking time was significantly shorter in the Xi group (5.0 vs. 3.0 min, respectively, $p=0.020$ ). Despite the significantly higher rate of the D2 lymph node dissection in the Xi group (76.2 vs. $34.6 \%$, respectively, $p=0.011$ ), the median console time showed no significant difference (57.5 vs. $56.0 \mathrm{~min}$, respectively, $p=0.404$ ). None of the other operative details or oncologic results showed any significant differences.

Conclusion: Although the enhanced anatomical access system of the Xi system, clinical outcomes did not be improved, except reducing docking time. Further investigations which can improve clinical outcomes are needed.

Keywords: Gastrectomy, Stomach neoplasms, Robotic surgical procedures

This is an Open Access article distributed under the terms of the Creative Commons Attribution Non-Commercial License (http:// creativecommons.org/licenses/by-nc/4.0/) which permits unrestricted non-commercial use, distribution, and reproduction in any medium, provided the original work is properly cited.

\section{INTRODUCTION}

With the increased incidence of early gastric cancer (EGC), the surgical treatment of gastric cancer has focused on quality of life, in which effort, minimally invasive surgery has been of particular interest. ${ }^{1-3}$ Since Kitano et al. introduced laparoscopy-assisted distal gastrectomy (LADG) in 1994, ${ }^{4}$ it has been widely performed in patients with EGC and, recently, has been expanded to those with advanced gastric cancer (AGC), ${ }^{5,6}$

In 1999, the first-generation da Vinci system (Intuitive Surgical Inc., Sunnyvale, CA), a robot incorporating the telepresence master/slave technique and four arms, offered surgeons three-dimensional (3D) vision as well as anti-tremor and
Received May 9, 2018

Revised June 22, 2018

Accepted July 3, 2018

Corresponding author

Kyo Young Song

Department of Surgery, Uijeongbu

St. Mary's Hospital, College of

Medicine, The Catholic University of

Korea, 271 Cheonbo-ro, Uijeongbu

11765, Korea

Tel: $+82-31-820-5069$

Fax: +82-31-847-2717

E-mail: skygs@catholic.ac.kr, skys9615@gmail.com
Copyright $\odot 2018$ The Journal of Minimally Invasive Surgery. All rights reserved.
Endo-Wrist motions. ${ }^{7-10}$ Since then, numerous da Vinci robotic surgeries have been performed in various surgical fields such as cholecystectomy, gastrointestinal surgery, colorectal surgery, gynecologic surgery, and urologic surgery. Additionally, in the field of gastric cancer surgery, robot-assisted gastrectomy (RAG) has been widely performed since its introduction in 2006. ${ }^{11}$

As the related technology has developed, improved generations of the system, starting with the da Vinci S in 2006, proceeding through the da Vinci Si in 2009 and then to the da Vinci Xi in 2014, have been released..$^{12}$ Application of various developed technologies such as skinny arms for flexible port placement, overhead-boom-mounted arms, universal 
arms and accessories for port hopping, and long-reach instruments subsequently have been introduced as special features of da Vinci Xi. These Xi iterations notwithstanding, there has been little comparative work done with respect to da Vinci Xi versus the older, S (the "da Vinci Standard") and/or Si generations. ${ }^{13,14}$

Here, we compare the da Vinci Xi with the da Vinci S for treatment (gastrectomy) of patients with gastric cancer. The specific aim of our study was to compare da Vinci Xi with da Vinci S and to identify the pros and cons of da Vinci Xi.

\section{MATERIALS AND METHODS}

\section{Patients and data collection}

A total of 47 patients who had undergone RAG (all performed by one gastric cancer specialist, Song KY) at Seoul St. Mary's Hospital between January 2015 and September 2016 were enrolled in this study. The selection was chronologically restricted, thusly, so as to reduce confounding factors related to the learning curves of the operating surgeon, assistants, and nurses. Among that cohort, 26 underwent gastrectomy by $d a$ Vinci S and 21 by da Vinci Xi. The S system had been used at our hospital from April 2009, the Xi system having been added from March 2016 (Fig. 1).

The patients' demographics, clinical and pathological characteristics, operative details, and short-term postoperative outcomes were collected retrospectively from the prospectively collected Gastric Cancer Patient Registry. Pathological stage was classified according to the 7th American Joint Cancer Committee (AJCC) TNM. ${ }^{15}$ The operative details included overall operation time, estimated blood loss (EBL), docking time, console time, resection type, lymph node (LN) dissection, and the reconstruction methods.

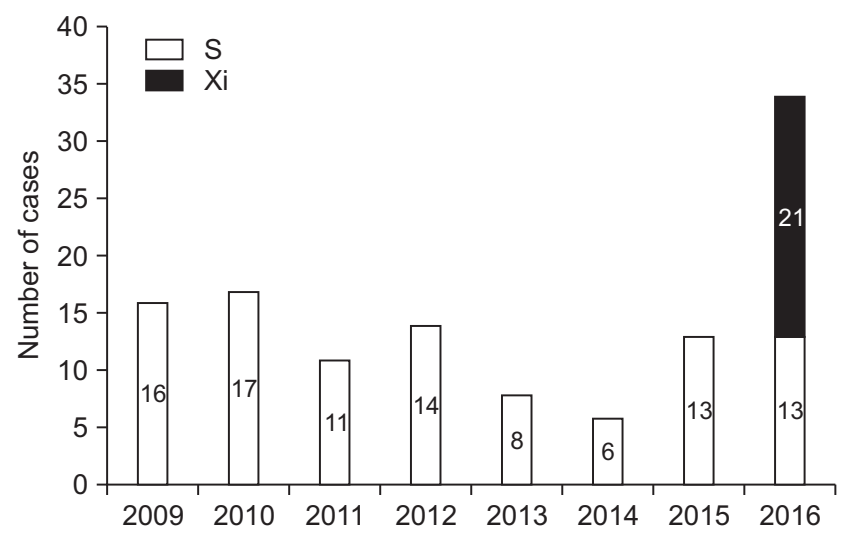

Fig. 1. Number of RAG procedures performed in Seoul St. Mary's Hospital by year.
This study was approved by the Institutional Review Board of the Ethics Committee of the College of Medicine, The Catholic University of Korea (KC16RISI0883). All of the patient records were anonymized and de-identified prior to the analysis.

\section{Operative details}

All of the patients underwent standard operations for gastric cancer, either total gastrectomy (TG) or distal gastrectomy (DG) with LN dissection, according to the 3rd Japanese Gastric Cancer Association (JGCA) treatment guidelines. ${ }^{16}$ The DG procedure followed is similar to that which has already been described. ${ }^{17}$ The patient was placed in the lithotomy position. da Vinci S and Xi were both configured as five-port systems. In the case of da Vinci S, a 12-mm umbilical port for the camera, three 8-mm ports for the robotic arms, and one 12$\mathrm{mm}$ port for the assistant were used; as for da Vinci Xi, four 8- $\mathrm{mm}$ ports for the robotic arms including the camera port, and one 12-mm port for the assistant were used (Fig. 2). The two 8-mm ports on the right side of the patient were used for Cadiere forceps and an ultrasonic shear, and the one on the left side was used for bipolar Maryland forceps.

Initially, after the port insertion and during the pre-console period, partial or total omentectomy, dissection of LN station 4sb,4d,6 and duodenal transection were laparoscopically performed. Then, after the robot docking and during the console period, the supra-pancreatic LN stations were dissected according to the 3rd JGCA treatment guidelines. ${ }^{16}$ After un-

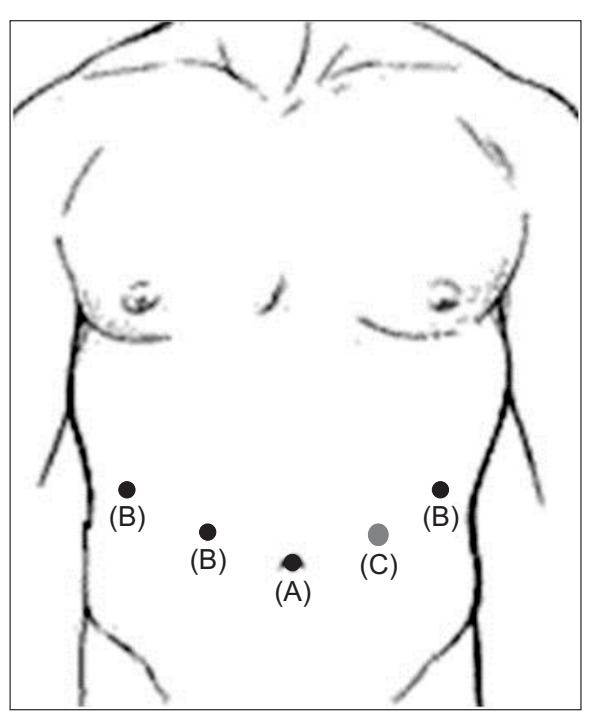

Fig. 2. Port sites for RAG: (A) umbilical camera port, 12-mm for $\mathrm{S}$, 8-mm for $\mathrm{Xi}_{\text {; }}$ (B) Three ports for robotic arms, 8-mm (C) port for assistant, 12$\mathrm{mm}$. 

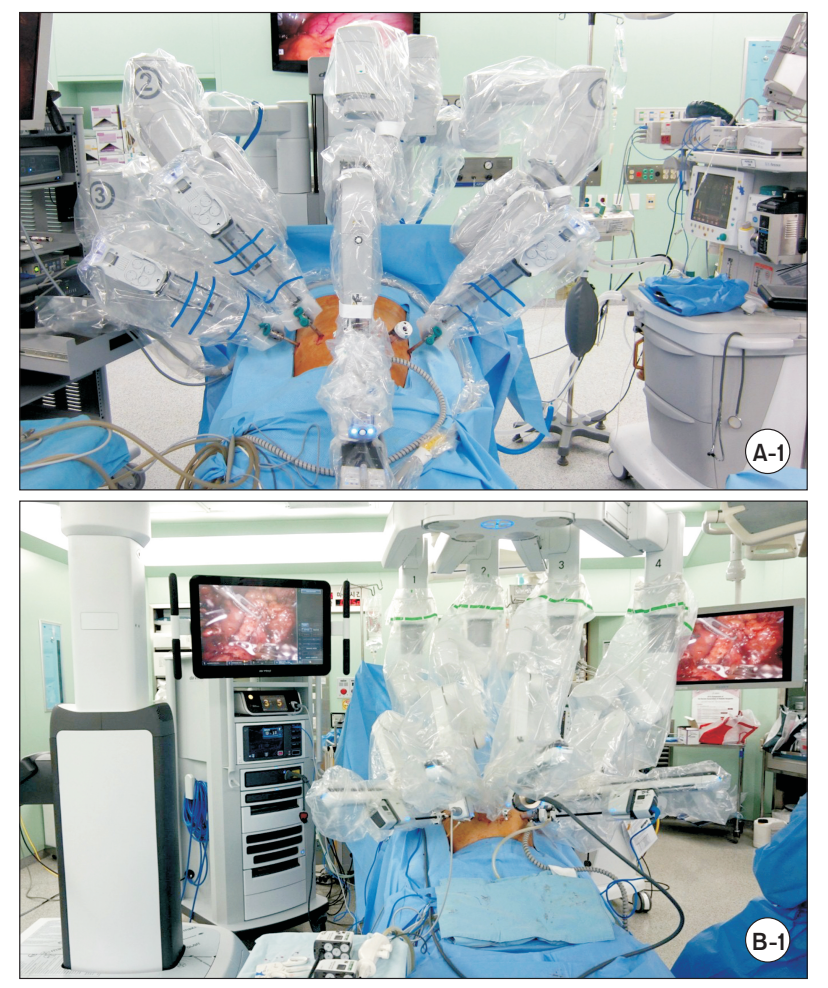

docking, gastric transection or esophageal resection was performed laparoscopically during the post-console period. The specimen was extracted through the extended umbilical port site. All of the modes of reconstruction were performed using circular or linear staplers intra-corporeally. With da Vinci S, the location of the robot arms is the cranial side of the patient, whereas with da Vinci Xi, it is the right side (Fig. 3).

Docking time was defined as the time necessary to move the robot into the surgical field and set all four robotic arms into their respective port sites. The console time was the actual time during which the operating surgeon performed at the robotic console.

\section{Statistical analysis}

The chi-square or Fisher's exact test was used to compare the categorical variables between the groups. The Student's ttest or Mann-Whitney U-test was used to compare the continuous variables. Statistical analyses were performed with the SPSS for Windows software (ver. 21.0; SPSS, Inc., Chicago, IL, USA). Values of $p<0.05$ were considered to indicate statistical significance.

\section{RESULTS}

Among the 47 patients, 26 were in the da Vinci S group and 21 in the da Vinci Xi group. There were no significant dif-

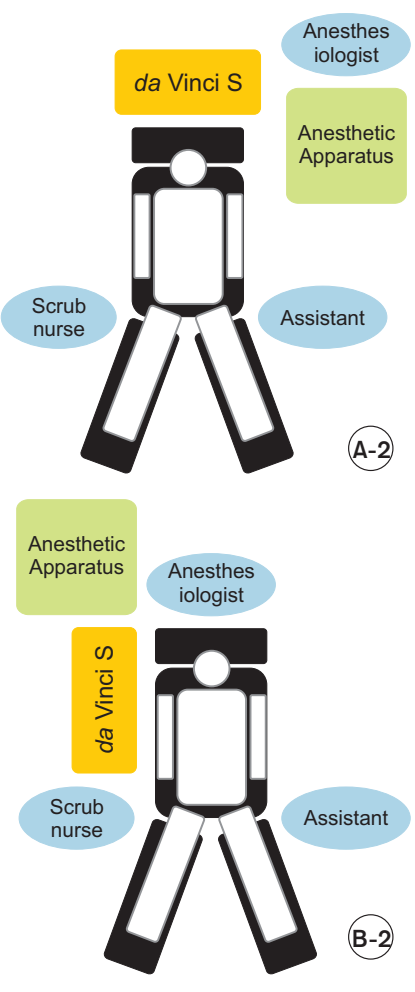

Fig. 3. Location of robot and operation team: (A-1) da Vinci S located overhead of patient, robotic arm for camera fixed to body of robot; (A-2) anesthesiologist steps aside from patient's head; (B-1) da Vinci Xi located on right side of patient, robotic arms connected to boom; (B-2) anesthesiologist stands above patient's head.

ferences in the clinical characteristics (i.e. age, sex, and BMI) between the groups. The pathological results likewise showed no significant differences (Table 1).

The operative details are shown in Table 2. As is apparent, there were no significant resection- or reconstructiontype differences $(p>0.05)$. D2 LN dissection was performed more frequently in the Xi group (76.2 vs. $34.6 \%, p=0.011$ ); also, median docking time was significantly shorter (3.0 vs. 5.0, $p=0.020$ ) and mean EBL was significantly smaller (43.8 vs. 59.2 $\mathrm{ml}, p=0.035)$. Neither operation time nor console time showed any significant difference (Table 2).

None of the short-term postoperative outcomes (e.g. postoperative complication rate, hospital stay, postoperative stay) showed a statistically significant difference between the two groups. There were no cases of mortality (Table 3). Grade III complication (according to the Clavien-Dindo classification ${ }^{18}$ ) was observed in one patient in each group.

\section{DISCUSSION}

RAG for patients with gastric cancer has been frequently performed in Korea. ${ }^{19}$ Since the first reported EGC use of RAG by Kakeji et al. in 2006, ${ }^{11}$ many surgeons from various centers have performed it, and not only for patients with EGC but also for those with AGC. Several reports have emphasized the benefits of robotic surgery, notwithstanding the ongoing controversy. ${ }^{20-22}$ We previously reported a significantly lower 
Table 1. Clinicopathological characteristics

\begin{tabular}{|c|c|c|c|}
\hline Characteristics & $\begin{array}{l}\text { da Vinci S } \\
(n=26)\end{array}$ & $\begin{array}{c}\text { da Vinci Xi } \\
\text { (n=21) }\end{array}$ & $p$ value \\
\hline Age, mean $\pm S D$ (years) & $52.7 \pm 10.9$ & $53.8 \pm 10.7$ & 0.738 \\
\hline Sex, n (male:female) & 11:15 & $12: 9$ & 0.473 \\
\hline $\mathrm{BMI}$, mean $\pm S D\left(\mathrm{~kg} / \mathrm{m}^{2}\right)$ & $23.9 \pm 2.7$ & $23.5 \pm 3.7$ & 0.648 \\
\hline Depth of invasion, $\mathrm{n}(\%)$ & & & 0.686 \\
\hline $\mathrm{T1}$ & $21(80.8)$ & $15(71.4)$ & \\
\hline T2 & $2(7.7)$ & $4(19.0)$ & \\
\hline T3 & $1(3.8)$ & 1 (4.8) & \\
\hline T4 & $2(7.7)$ & 1 (4.8) & \\
\hline Lymph node metastasis, n (\%) & & & 0.584 \\
\hline NO & $23(88.5)$ & $16(76.2)$ & \\
\hline N1 & $2(7.7)$ & $3(14.3)$ & \\
\hline N2 & $1(3.8)$ & $1(4.8)$ & \\
\hline N3 & $0(0)$ & $1(4.8)$ & \\
\hline $\begin{array}{l}\text { Number of retrieved LNs, } \\
\mathrm{n}(\text { mean } \pm \text { SD) }\end{array}$ & $42.5 \pm 14.1$ & $45.4 \pm 12.0$ & 0.460 \\
\hline $\begin{array}{l}\text { Number of metastatic LNs, } \\
n(\text { mean } \pm \text { SD) }\end{array}$ & $0.3 \pm 1.0$ & $1.3 \pm 3.1$ & 0.174 \\
\hline Pathological stage* ${ }^{*}|\%|$ & & & 0.653 \\
\hline IA & $19(73.1)$ & $14(66.7)$ & \\
\hline IB & $2(7.7)$ & $3(14.3)$ & \\
\hline$\| \mathrm{A}$ & $3(11.5)$ & $1(4.8)$ & \\
\hline$\| B$ & $2(7.7)$ & 2 (9.5) & \\
\hline IIIC & $0(0)$ & $1(4.8)$ & \\
\hline
\end{tabular}

incidence of postoperative pancreatic fistula for robotic than for laparoscopic surgery. ${ }^{23}$

In the 1950s, the telepresence robotic technique was introduced for management of dangerous substances such as nuclear materials. In the medical field, the first da Vinci system, known today as the "da Vinci Standard," was introduced by Intuitive Surgical Inc (Sunnyvale, CA, USA) in 1999. This system had been designed to overcome the limitations of traditional laparoscopy by giving surgeons 3D vision and Endo-Wrist motion. After the several generations, the latestgeneration da Vinci, the Xi system, released in 2014, boasts multi-quadrant access (anatomical access) and crystal-clear HD vision. The tangible benefits of such techniques and fea-
Table 2. Operation details

\begin{tabular}{|c|c|c|c|}
\hline Characteristics & $\begin{array}{c}\text { da Vinci S } \\
(n=26)\end{array}$ & $\begin{array}{c}\text { da Vinci } X i \\
(n=21)\end{array}$ & $p$ value \\
\hline Resection, n (\%) & & & 1.000 \\
\hline $\mathrm{TG}$ & $2(7.7)$ & $2(9.5)$ & \\
\hline $\mathrm{DG}$ & 24 (92.3) & 19 (90.5) & \\
\hline LN dissection, n $(\%)$ & & & 0.011 \\
\hline $\mathrm{D} 1+$ & $17(65.4)$ & $5(23.8)$ & \\
\hline D2 & $9(34.6)$ & $16(76.2)$ & \\
\hline Reconsturction, n (\%) & & & 0.432 \\
\hline$B-1$ & $5(19.2)$ & $5(23.8)$ & \\
\hline B-II & $18(69.2)$ & $11(52.4)$ & \\
\hline R-Y & $3(11.5)$ & $5(23.8)$ & \\
\hline $\begin{array}{l}\text { Docking time, } \\
\text { median (IQR) (min) }\end{array}$ & $5.0(3.0 \sim 7.0)$ & $3.0(2.0 \sim 4.0)$ & 0.020 \\
\hline $\begin{array}{l}\text { Console time, } \\
\text { median (IQR) (min) }\end{array}$ & $57.5(38.0 \sim 77.0)$ & $56.0(52.0 \sim 75.0)$ & 0.404 \\
\hline $\begin{array}{l}\text { Operation time, } \\
\text { mean } \pm \text { SD (min) }\end{array}$ & $195.8 \pm 36.7$ & $204.3 \pm 36.3$ & 0.431 \\
\hline $\mathrm{EBL}$, mean $\pm \mathrm{SD}(\mathrm{ml})$ & $59.2 \pm 29.9$ & $43.8 \pm 18.3$ & 0.035 \\
\hline
\end{tabular}

$\mathrm{TG}=$ total gastrectomy; $\mathrm{DG}=$ distal gastrectomy; $\mathrm{LN}=$ lymph node; $\mathrm{IQR}=$ interquartile range; $\mathrm{SD}=$ standard deviation; $\mathrm{EBL}=$ estimated blood loss.

Table 3. Short-term postoperative outcomes

\begin{tabular}{|c|c|c|c|}
\hline Outcomes & $\begin{array}{c}\text { da Vinci S } \\
(n=26)\end{array}$ & $\begin{array}{c}\text { da Vinci Xi } \\
(\mathbf{n}=21)\end{array}$ & $p$ value \\
\hline Morbidity*, n $(\%)$ & & & 0.826 \\
\hline No complications & $21(80.8)$ & $17(81.0)$ & \\
\hline Grade I & 1 (3.8) & $0(0)$ & \\
\hline Grade II & $3(11.5)$ & $3(14.3)$ & \\
\hline Grade III & $1(3.8)$ & $1(4.8)$ & \\
\hline $\begin{array}{l}\text { Hospital stay, days, median } \\
\text { (IQR) }\end{array}$ & $9(9 \sim 10)$ & $9(9 \sim 9)$ & 0.068 \\
\hline $\begin{array}{l}\text { Postoperative stay, days, } \\
\text { median (IOR) }\end{array}$ & $7(7 \sim 8)$ & $7(7 \sim 7)$ & 0.068 \\
\hline
\end{tabular}

$\mathrm{IQR}=$ interquartile range; ${ }^{*}$ Within 30 postoperative days.

tures for actual surgeries, though, have remained unclear. $\mathrm{Oz}^{-}$ ben et al. reported that despite the da Vinci Xi system's shorter console time (owed to its ease of use), its rectal cancer surgery results were mostly similar to those for $\mathrm{da}$ Vinci $\mathrm{Si}^{13} \mathrm{Ad}^{-}$ 
ditionally, Kim et al. reported that da Vinci offers benefits in its anatomical access technique in trans-oral robotic surgery. ${ }^{14}$ However, to our best knowledge, there has been no comparative report on the various generations of $d a$ Vinci robots as they are applied to the treatment of gastric cancer.

Our center has employed the da Vinci S system since 2009 in gynecologic-, urologic-, thyroid-, colorectal- and gastrointestinal-surgery settings. Additionally, we have used da Vinci Xi since March 2016. A total of 119 RAG procedures for patients with gastric cancer have been performed since April 2009, including $98 \mathrm{~S}$ cases and 21 Xi cases. At present, RAG is performed with either the da Vinci S or Xi systems at our center.

Among the key findings of the present study was the fact that for the Xi group of patients, the docking time had been decreased. There are several possible explanations for this. The skinny arms might lessen the effects of extracorporeal crushing between them. What also must be considered is the auto-targeting system's optimal positioning for the targeted organ. The boom, which is the connection center of the arms, can facilitate anatomical access via its rotating and elevating motions. Certainly too, this anatomical access had another benefit, this one related to the difference in the positions of the da Vinci S and Xi robots. da Vinci S approaches from the patient's cranial side, which necessitates that the anesthesiologist move from the optimal position at the patient's head to the patient's left side. This setup incurs additional labor for the anesthesiologist (Fig. 3A). By contrast, da Vinci Xi approaches from the patient's right side, and the boom rotates for better positioning relative to the patient. Thus, the anesthesiologist can be positioned on the cranial side of the patient, from where any emergency can easily be managed (Fig. 3B).

Multi-quadrant accessibility, another known benefit of the boom, has been reported to be useful in colorectal cancer surgery, which entails multi-quadrant dissection. ${ }^{13}$ In the present study, we performed only suprapancreatic LN dissection using robot. And that procedure is mainly performed on the patient's left-upper quadrant or epigastric area. It might be an important reason for not showing significant benefits of $\mathrm{Xi}$ system. However, although we did not show it in our results, the advantage of multi-quadrant accessibility can be found if the operation was proceed with the robot form the beginning to the end.

In gastric cancer surgery, LN dissection usually is performed during the console period. In the present study, although D2 LN dissection was performed more often in the $\mathrm{Xi}$ group, the console time showed no significant difference between the two groups. Moreover, ELB was slightly smaller in the Xi group (Table 2). This might have resulted from the improved $\mathrm{HD}$ vision and instrumentation.
The FireFly system, which can detect indocyanine green (ICG) and present its findings as fluorescence images, was applied to the da Vinci Si system in 2009. Recently, in the EGCsurgical setting, ICG-fluorescence image-guided surgery by peritumoral ICG injection through intraoperative or preoperative endoscopy has been performed for detection of sentinel LNs. ${ }^{24,25}$ ICG-fluorescence image-guided surgery for EGC has been performed at our center several times as well. Whereas its usefulness for sentinel-LN detection has not yet been determined, it is in fact beneficial for fine-node dissection and tumor localization in EGC patients.

According to our overall results, neither the TNM stage of the patients nor the number of retrieved LNs showed any significant difference between the da Vinci S and Xi systems (Table 1). Moreover, there were no significant differences in complication rate, hospital stay, or postoperative stay either (Table 3).

The present study has several limitations. First, it was a retrospective study with a limited number of cases. Second, the choice of the robot type was made by the operating surgeon without randomization; thus, selection bias could have been incurred. Third, although there was only one surgeon, the assistant and scrub-nurse were changed several times. Lastly, only suprapancreatic LN dissection was performed using robot. Although the suprapancreatic LN dissection is the most important procedure during gastrectomy, it is limited to present the real robotic gastrectomy procedure.

In summary, there were no significant differences between the da Vinci S and the Xi systems in operation time or oncologic features. The sole objective distinction was the significantly shorter docking time of da Vinci Xi. Notwithstanding, further investigation is necessary, particularly respecting the newer incorporated technologies such as the improved HD vision and the developed instruments such as the linear staplers with Endo-Wrist motion.

\section{ACKNOWLEDGMENTS}

The authors deeply appreciate the great help provided by Kyung Nam Lee, the charge nurse of the robotic operating room in Seoul St. Mary's Hospital, The Catholic University of Korea.

\section{REFERENCES}

1) Oh CM, Won YJ, Jung KW, et al. Cancer Statistics in Korea: Incidence, Mortality, Survival, and Prevalence in 2013. Cancer Res Treat 2016;48:436-450.

2) Kim YW, Baik YH, Yun YH, et al. Improved quality of life outcomes after laparoscopy-assisted distal gastrectomy for early gas- 
tric cancer: results of a prospective randomized clinical trial. Ann Surg 2008;248:721-727.

3) Pyo JH, Lee H, Min BH, et al. Long-Term Outcome of Endoscopic Resection vs. Surgery for Early Gastric Cancer: A Noninferiority-Matched Cohort Study. Am J Gastroenterol 2016;111: 240-249.

4) Kitano S, Iso Y, Moriyama M, Sugimachi K. Laparoscopy-assisted Billroth I gastrectomy. Surg Laparosc Endosc 1994;4:146-148.

5) Hur H, Lee HY, Lee HJ, et al. Efficacy of laparoscopic subtotal gastrectomy with D2 lymphadenectomy for locally advanced gastric cancer: the protocol of the KLASS-02 multicenter randomized controlled clinical trial. BMC Cancer 2015;15:355.

6) Kim HI, Hur H, Kim YN, et al. Standardization of D2 lymphadenectomy and surgical quality control (KLASS-02-QC): a prospective, observational, multicenter study [NCT01283893]. BMC Cancer 2014;14:209.

7) Sung GT, Gill IS. Robotic laparoscopic surgery: a comparison of the DA Vinci and Zeus systems. Urology 2001;58:893-898.

8) Kim VB, Chapman WH, Albrecht RJ, et al. Early experience with telemanipulative robot-assisted laparoscopic cholecystectomy using da Vinci. Surg Laparosc Endosc Percutan Tech 2002;12:33-40.

9) Koh DC, Tsang CB, Kim SH. A new application of the four-arm standard da Vinci(R) surgical system: totally robotic-assisted leftsided colon or rectal resection. Surg Endosc 2011;25:1945-1952.

10) Senapati S, Advincula AP. Surgical techniques: robot-assisted laparoscopic myomectomy with the da $\operatorname{Vinci}((\mathrm{R}))$ surgical system. J Robot Surg 2007;1:69-74.

11) Kakeji $Y$, Konishi $K$, Ieiri $S$, et al. Robotic laparoscopic distal gastrectomy: a comparison of the da Vinci and Zeus systems. Int J Med Robot 2006;2:299-304.

12) Intutuve Surgical. History and Products of da Vinci ${ }^{\circledR}$ [Internet]. c2016 [cited on 2018 Apr. 27]. Available from: https://www.intuitive.com/.

13) Ozben V, Cengiz TB, Atasoy D, et al. Is da Vinci Xi Better than da Vinci Si in Robotic Rectal Cancer Surgery? Comparison of the 2 Generations of da Vinci Systems. Surg Laparosc Endosc Percutan Tech 2016;26:417-423.

14) Kim DH, Kim H, Kwak S, et al. The Settings, Pros and Cons of the New Surgical Robot da Vinci Xi System for Transoral Robotic Surgery (TORS): A Comparison With the Popular da Vinci Si System. Surg Laparosc Endosc Percutan Tech 2016;26:391-396.

15) Washington K. 7th edition of the AJCC cancer staging manual: stomach. Ann Surg Oncol 2010;17:3077-3079.

16) Japanese Gastric Cancer Association. Japanese gastric cancer treatment guidelines 2010 (ver. 3). Gastric Cancer 2011;14:113123.

17) Lee HH, Hur H, Jung H, Jeon HM, Park CH, Song KY. Robotassisted distal gastrectomy for gastric cancer: initial experience. Am J Surg 2011;201:841-845.

18) Clavien PA, Barkun J, de Oliveira ML, et al. The Clavien-Dindo classification of surgical complications: five-year experience. Ann Surg 2009;250:187-196.

19) Song J, Kang WH, Oh SJ, Hyung WJ, Choi SH, Noh SH. Role of robotic gastrectomy using da Vinci system compared with laparoscopic gastrectomy: initial experience of 20 consecutive cases. Surg Endosc 2009;23:1204-1211.

20) Kim YM, Son T, Kim HI, Noh SH, Hyung WJ. Robotic D2 Lymph Node Dissection During Distal Subtotal Gastrectomy for Gastric Cancer: Toward Procedural Standardization. Ann Surg Oncol 2016;23:2409-2410.

21) Suda K, Nakauchi M, Inaba K, Ishida Y, Uyama I. Robotic surgery for upper gastrointestinal cancer: Current status and future perspectives. Dig Endosc 2016;28:701-713.

22) Quijano Y, Vicente E, Ielpo B, et al. Full robot-assisted gastrectomy: surgical technique and preliminary experience from a single center. J Robot Surg 2016;10:297-306.

23) Seo HS, Shim JH, Jeon HM, Park CH, Song KY. Postoperative pancreatic fistula after robot distal gastrectomy. J Surg Res 2015; 194:361-366.

24) Kinami S, Oonishi T, Fujita J, et al. Optimal settings and accuracy of indocyanine green fluorescence imaging for sentinel node biopsy in early gastric cancer. Oncol Lett 2016;11:4055-4062.

25) Mitsumori N, Nimura H, Takahashi N, et al. Sentinel lymph node navigation surgery for early stage gastric cancer. World J Gastroenterol 2014;20:5685-5693. 